\title{
Black string in dRGT massive gravity
}

\author{
Lunchakorn Tannukij ${ }^{1,2,3, a}$, Pitayuth Wongjun ${ }^{3,4, b}$, Suchant G. Ghosh ${ }^{5,6, c}$ \\ ${ }^{1}$ Department of Physics, Faculty of Science, Mahidol University, Bangkok 10400, Thailand \\ 2 Department of Physics, Hanyang University, Seoul 133-891, Korea \\ ${ }^{3}$ The Institute for Fundamental Study, Naresuan University, Phitsanulok 65000, Thailand \\ 4 Thailand Center of Excellence in Physics, Ministry of Education, Bangkok 10400, Thailand \\ ${ }^{5}$ Centre of Theoretical Physics, Jamia Millia Islamia, New Delhi 110025, India \\ ${ }^{6}$ Astrophysics and Cosmology Research Unit, School of Mathematical Sciences, University of Kwazulu-Natal, Private Bag 54001, Durban 4000, \\ South Africa
}

Received: 1 February 2017 / Accepted: 28 November 2017 / Published online: 8 December 2017

(C) The Author(s) 2017. This article is an open access publication

\begin{abstract}
We present a cylindrically symmetric solution, both charged and uncharged, which is known as a black string solution to the nonlinear ghost-free massive gravity found by de Rham, Gabadadze, and Tolley (dRGT). This "dRGT black string" can be thought of as a generalization of the black string solution found by Lemos. Moreover, the dRGT black string solution includes other classes of black string solution such as the monopole-black string ones since the graviton mass contributes to the global monopole term as well as the cosmological-constant term. To investigate the solution, we compute mass, temperature, and entropy of the dRGT black string. We found that the existence of the graviton mass drastically affects the thermodynamics of the black string. Furthermore, the Hawking-Page phase transition is found to be possible for the dRGT black string as well as the charged dRGT black string. The dRGT black string solution is thermodynamically stable for $r>r_{c}$ with negative thermodynamical potential and positive heat capacity while it is unstable for $r<r_{c}$ where the potential is positive.
\end{abstract}

\section{Introduction}

In general relativity (GR), where the corresponding graviton is a massless spin-2 particle, is the current description of gravitation in physics and has importantly astrophysical implications. One of the alternating theory of gravity, which follows a very simple idea of generalization to GR, is known as massive gravity where interaction terms, corresponding to graviton mass, are added into GR. The result of such an intro-

\footnotetext{
a e-mail: 1_tannukij@hotmail.com

be-mail: pitbaa@gmail.com

c e-mail: sgghosh@gmail.com
}

duction is that the model of gravity is modified significantly at a large scale so that, from the cosmological point of view, a system like our universe can enter the cosmic accelerating expansion phase. Not only that; the gravity at smaller scale is not altered much by such a modification to ensure the same predictions as GR makes.

However, adding generic mass terms for the graviton on given background usually brings about various instabilities and ghosts for the gravitational theories. To avoid the appearance of the ghost in massive gravity suggested by Boulware and Deser [1], the set of possible interaction terms is constructed accordingly by de Rham, Gabadadze and Tolley (dRGT) [2,3]. In particular, the interaction terms are constructed in a specific way to ensure that the corresponding equations of motion are at most second order differential equations so that there is no ghost field. The allowed interaction terms for the four-dimensional theory consist of three kinds of combination; the quadratic, the cubic, and the quartic orders of the metric. Unfortunately, such nonlinearities involve complexities in the calculation as a price to pay for eliminating the ghost and usually make it cumbersome to find an exact solution. Nevertheless, there have been plenty interesting approaches to tackle the complexity to obtain spherical symmetric solutions in massive gravity theories [4-26]. In particular, it was found by Vegh [4] that a spherically symmetric black hole with a Ricci flat horizon is a solution to the four-dimensional massive gravity and then the solution was studied in the aspect of thermodynamical properties and phase transition structure $[5,6]$. The spherically symmetric solutions for dRGT were also considered in $[9,10]$. The extension of the solution in terms of electric charge was found in $[11,12]$. Moreover, the bi-gravity extension of the solution was found in [13] which covers the previously known spherically symmetric solutions (see [14-16] for reviews on black 
holes in massive gravity). Recently, other extensions of the dRGT massive gravity solution were explored [27-36].

In astrophysics, it is commonly known that black holes can be created by the gravitational collapse of massive stars. However, the famous Thorne [37] hoop conjecture states that gravitational collapse of a massive star will yield a black hole only if the mass $M$ is compressed to a region with circumference $C<4 \pi M$ in all directions. If the hoop conjecture is true, cylindrical matter will not form a black hole. However, the hoop conjecture was given for a spacetime with a zero cosmological constant. When a negative cosmological constant is introduced, the spacetime will become an asymptotically anti-de Sitter spacetime. Indeed, Lemos [38] has shown a possibility of cylindrical black holes from the gravitational collapse with cylindrical matter distribution in an anti-de Sitter spacetime, violating in this way the hoop conjecture. This cylindrically static black hole solutions in an anti-de Sitter spacetime are called black strings. The pioneering work on the black string is due to Lemos [39-41], and soon its charged and rotating [42] counterpart were also obtained.

The main purpose of this paper is to present an exact black string solution, including its generalization to the charge case in dRGT massive gravity, and also to discuss their thermodynamical properties with a focus on thermodynamic stability. The possibility to obtain a Hawking-Page phase transition is explored. We use units which fix the speed of light and the gravitational constant via $G=c=1$, and use the metric signature $(-,+,+,+)$.

\section{2 dRGT massive gravity}

In this section we review an important ingredient of dRGT massive gravity theory, which is a well-known nonlinear generalization of a massive gravity and is free of the BoulwareDeser ghost by incorporating higher order interaction terms into the Lagrangian. The dRGT massive gravity action is the well-known Einstein-Hilbert action plus suitable nonlinear interaction terms as given by [3]

$S=\int \mathrm{d}^{4} x \sqrt{-g} \frac{1}{2}\left[R(g)+m_{g}^{2} \mathcal{U}(g, f)\right]$,

where $R$ is the Ricci scalar and $\mathcal{U}$ is a potential for the graviton which modifies the gravitational sector with the parameter $m_{g}$ interpreted as the graviton mass. The effective potential $\mathcal{U}$ in four-dimensional spacetime is given by

$\mathcal{U}\left(g, \phi^{a}\right)=\mathcal{U}_{2}+\alpha_{3} \mathcal{U}_{3}+\alpha_{4} \mathcal{U}_{4}$,

where $\alpha_{3}$ and $\alpha_{4}$ are dimensionless free parameters of the theory. The terms $\mathcal{U}_{2}, \mathcal{U}_{3}$ and $\mathcal{U}_{4}$ can be written in terms of the physical metric $g_{\mu \nu}$ and the reference (or fiducial) metric $f_{\mu \nu}$ as

$$
\begin{aligned}
& \mathcal{U}_{2} \equiv[\mathcal{K}]^{2}-\left[\mathcal{K}^{2}\right] \\
& \mathcal{U}_{3} \equiv[\mathcal{K}]^{3}-3[\mathcal{K}]\left[\mathcal{K}^{2}\right]+2\left[\mathcal{K}^{3}\right] \\
& \mathcal{U}_{4} \equiv[\mathcal{K}]^{4}-6[\mathcal{K}]^{2}\left[\mathcal{K}^{2}\right]+8[\mathcal{K}]\left[\mathcal{K}^{3}\right]+3\left[\mathcal{K}^{2}\right]^{2}-6\left[\mathcal{K}^{4}\right]
\end{aligned}
$$

where

$\mathcal{K}_{v}^{\mu}=\delta_{v}^{\mu}-\sqrt{g^{\mu \nu} f_{\mu \nu}}$,

where the rectangular brackets denote the traces, namely $[\mathcal{K}]=\mathcal{K}_{\mu}^{\mu}$ and $\left[\mathcal{K}^{n}\right]=\left(\mathcal{K}^{n}\right)_{\mu}^{\mu}$. Note that we choose the unitary gauge in our consideration [4]. One can see that there are no kinetic terms for the fiducial metric. It seems to play the role of the Lagrange multiplier to help us to construct the suitable form of the mass term. Conveniently, we redefine the two parameters $\alpha_{3}$ and $\alpha_{4}$ of the graviton potential in Eq. (2) by introducing two new parameters $\alpha$ and $\beta$, as follows:

$\alpha_{3}=\frac{\alpha-1}{3}, \quad \alpha_{4}=\frac{\beta}{4}+\frac{1-\alpha}{12}$.

The equation of motion of the theory can be obtained by varying the action with respect to the dynamical metric $g_{\mu \nu}$ as

$G_{\mu \nu}+m_{g}^{2} X_{\mu \nu}=0$,

where $X_{\mu \nu}$ is obtained by varying the potential term with respect to $g_{\mu \nu}$ known as the effective energy-momentum tensor. This effective energy-momentum tensor can be written explicitly as

$$
\begin{aligned}
X_{\mu \nu}= & \mathcal{K}_{\mu \nu}-\mathcal{K} g_{\mu \nu}-\alpha\left(\mathcal{K}_{\mu \nu}^{2}-\mathcal{K} \mathcal{K}_{\mu \nu}+\frac{\mathcal{U}_{2}}{2} g_{\mu \nu}\right) \\
& +3 \beta\left(\mathcal{K}_{\mu \nu}^{3}-\mathcal{K} \mathcal{K}_{\mu \nu}^{2}+\frac{\mathcal{U}_{2}}{2} \mathcal{K}_{\mu \nu}-\frac{\mathcal{U}_{3}}{6} g_{\mu \nu}\right),
\end{aligned}
$$

and it obeys a constraint by using the Bianchi identities as follows:

$\nabla^{\mu} X_{\mu \nu}=0$,

where $\nabla^{\mu}$ denotes the covariant derivative which is compatible with $g_{\mu \nu}$. In the next section, we will find the solution to this equation of motion by imposing the static and axial symmetry.

\section{Black string solution in dRGT massive gravity}

In this section, we will look for a static and axially symmetric solution of the modified Einstein equations (8) with the following physical metric ansatz:

$\mathrm{d} s^{2}=-n(r) \mathrm{d} t^{2}+2 d(r) \mathrm{d} t \mathrm{~d} r+\frac{\mathrm{d} r^{2}}{f(r)}+L(r)^{2} \mathrm{~d} \Omega^{2}$, 
and with the Minkowski flat fiducial metric,

$\mathrm{d} s^{2}=-\mathrm{d} t^{2}+\mathrm{d} r^{2}+r^{2} \mathrm{~d} \Omega^{2}$.

Here $\mathrm{d} \Omega^{2}=\mathrm{d} \varphi^{2}+\alpha_{g}^{2} \mathrm{~d} z^{2}$ is a metric on the 2-D surface. The 2-D surface is chosen to be compatible with the black string solution $[40,43]$. The solutions in this ansatz can be classified into two branches: $d(r)=0$ or $L(r)=l_{0} r$, where $l_{0}$ is a constant, in terms of the parameters $\alpha$ and $\beta$ as

$l_{0}=\frac{\left(\alpha+3 \beta \pm \sqrt{\alpha^{2}-3 \beta}\right)}{2 \alpha+3 \beta+1}$.

From this stage, it is convenient to investigate the branch $d=0$. Even though we choose to investigate the simple branch, the equation is still difficult to investigate analytically. Since the fiducial metric seems to play the role of a Lagrange multiplier to eliminate the BD ghost, one can choose an appropriate form to simplify the calculation. Indeed, the suitable form of the fiducial metric significantly provides the analytical form of the physical metric, for example [4] for a spherically symmetric solution and [44] for a cosmological solution. In the present work, we choose the fiducial metric to be

$f_{\mu \nu}=\operatorname{diag}\left(0,0, h(r)^{2}, \alpha_{f}^{2} h(r)^{2}\right)$,

where $h(r)$ is an arbitrary function. The line element of the corresponding physical metric in this branch can be written as

$\mathrm{d} s^{2}=-n(r) \mathrm{d} t^{2}+\frac{\mathrm{d} r^{2}}{f(r)}+L(r)^{2} \mathrm{~d} \Omega^{2}$.

From this ansatz, the components of the Einstein tensor can be written as

$$
\begin{aligned}
G_{t}^{t} & =\frac{L f^{\prime} L^{\prime}+2 f L L^{\prime \prime}+f L^{\prime 2}}{L^{2}}, \\
G_{r}^{r} & =\frac{f L^{\prime}\left(n L^{\prime}+L n^{\prime}\right)}{L^{2} n}, \\
G_{\varphi}^{\varphi}=G_{z}^{z} & =\frac{n f^{\prime}\left(2 n L^{\prime}+L n^{\prime}\right)+f\left(4 n^{2} L^{\prime \prime}+2 n L^{\prime} n^{\prime}+2 L n n^{\prime \prime}-L n^{\prime 2}\right)}{4 L n^{2}} .
\end{aligned}
$$

Computing the effective energy-momentum tensor in Eq. (9) with this ansatz, the tensor $X_{\mu \nu}$ can be written as

$$
\begin{aligned}
& X_{t}^{t}=X_{r}^{r}=\frac{h \alpha_{f}}{L \alpha_{g}}-\frac{3 L-h}{L}+\alpha\left(\frac{h \alpha_{f}(2 L-h)}{L^{2} \alpha_{g}}-\frac{3 L-2 h}{L}\right) \\
& +\beta\left(\frac{3 h \alpha_{f}(L-h)}{L^{2} \alpha_{g}}-\frac{3(L-h)}{L}\right), \\
& X_{\varphi}^{\varphi}=\frac{h \alpha_{f}}{L \alpha_{g}}-3+\alpha\left(\frac{2 h \alpha_{f}}{L \alpha_{g}}-3\right)+\beta\left(\frac{3 h \alpha_{f}}{L \alpha_{g}}-3\right) \text {, } \\
& X_{z}^{z}=-\left(\frac{h-3 L}{L}+\frac{\alpha(2 h-3 L)}{L}+\frac{3 \beta(h-L)}{L}\right) .
\end{aligned}
$$

By substituting these components into the modified equation in Eq. (8) and then using the equations for the $(t, t)$ and $(r, r)$ components, we found the constraint

$\frac{\mathrm{d}}{\mathrm{d} r}\left(\frac{f L^{\prime 2}}{n}\right)=0$.

In order to obtain the black hole solution with $f=n$, the function $L(r)$ must be proportional to $r$. Therefore, we can set $L=r$ for the following investigation. By using this setting, we have only two independent functions, $f$ and $h$. The two independent equations for these two functions are the conservation of the energy-momentum tensor in Eq. (10) and the $(t, t)$ component of the modified Einstein equation in Eq. (8), which can be expressed, respectively, as

$$
\begin{aligned}
& \frac{h^{\prime}\left(r(1+2 \alpha+3 \beta)\left(\alpha_{f}+\alpha_{g}\right)-2 h \alpha_{f}(\alpha+3 \beta)\right)}{\alpha_{g} r^{2}}=0 \\
& \frac{r f^{\prime}+f}{r^{2}}=-m_{g}^{2}\left(\frac{h \alpha_{f}}{\alpha_{g} r}-\frac{3 r-h}{r}\right. \\
& +\alpha\left(\frac{h \alpha_{f}(2 r-h)}{\alpha_{g} r^{2}}-\frac{3 r-2 h}{r}\right) \\
& \left.+\beta\left(\frac{3 h \alpha_{f}(r-h)}{\alpha_{g} r^{2}}-\frac{3(r-h)}{r}\right)\right)
\end{aligned}
$$

From Eq. (23), two exact solutions of $h$ can be written as

$h(r)=\frac{r(1+2 \alpha+3 \beta)\left(\alpha_{f}+\alpha_{g}\right)}{2 \alpha_{f}(\alpha+3 \beta)}$,

$h(r)=h_{0}=$ constant,

and with these solutions of $h(r)$, Eq. (24) admits the following two solutions:

$$
\begin{aligned}
f_{1}(r)= & \left(-\frac{m_{g}^{2}\left(1+\alpha+\alpha^{2}-3 \beta\right)}{3(\alpha+3 \beta)}\right) r^{2}-\frac{b}{\alpha_{g} r}, \\
f_{2}(r)= & -\frac{b}{\alpha_{g} r}+m_{g}^{2}\left((1+\alpha+\beta) r^{2}\right. \\
& \left.-h_{0}(1+2 \alpha+3 \beta) r+h_{0}^{2}(\alpha+3 \beta)\right),
\end{aligned}
$$

where $b$ is an integration constant. Note that we have used the component $(\varphi, \varphi)$ of the modified Einstein equation in Eq. (8) to obtain the constraint $\alpha_{f}=\alpha_{g}$. The first solution in Eq. (27) coincides with the black string solution in general relativity $[39,40,43]$ with an effective cosmological constant $\Lambda$ as

$\Lambda=-\frac{m_{g}^{2}\left(1+\alpha+\alpha^{2}-3 \beta\right)}{3(\alpha+3 \beta)}$.

Note that in $[39,40,43]$, the constant $\alpha_{g}^{2}$ is chosen to be $\alpha_{g}^{2}=$ $-\Lambda / 3$, and

$\alpha_{g}^{2}=\frac{m_{g}^{2}\left(1+\alpha+\alpha^{2}-3 \beta\right)}{(\alpha+3 \beta)}$. 

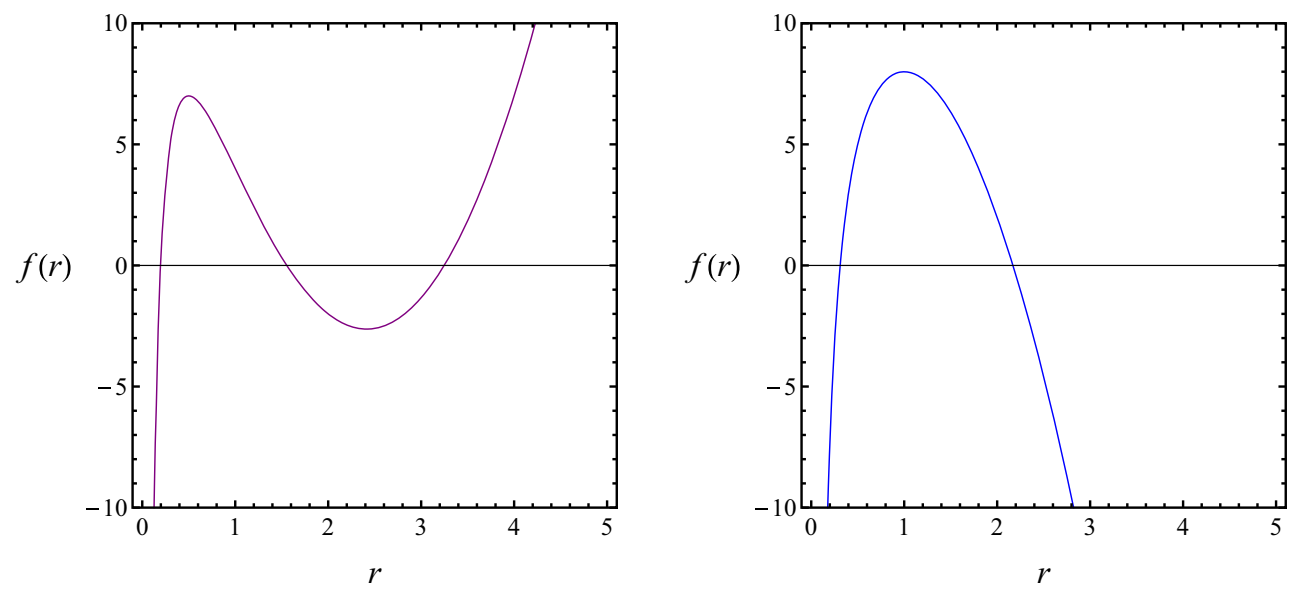

Fig. 1 The left panel shows the horizon structure of the AdS solution for specific values of the parameters where $\alpha_{m}^{2}=4, c_{0}=6, c_{1}=5$. The right panel shows the horizon structure of the dS solution for the value of the parameters as $\alpha_{m}^{2}=-4, c_{0}=-3, c_{1}=1$

The integration constant $b$ can be obtained by using the solutions in the Newtonian limit which can be expressed as $b=4 M$, where $M$ is the ADM mass per unit length in $z$ direction. Mathematically, the solution (27) is exactly the same as the one in general relativity which is already widely investigated. Moreover, its charged and rotating counterparts, and their properties including thermodynamics, have also widely been investigated [37-42]. Although the solution (27) coincides mathematically with that of Lemos [39,40,43], the dRGT solution naturally generates the cosmologicalconstant-like term from the graviton mass while for Lemos' black string solution [39] the cosmological constant is introduced by hand. Hence, the properties of the solution (27) can be analyzed as in the previously mentioned cases, and hence we shall not address them here. The second solution in Eq. (28) is new. We can redefine the variables and parameters as follows:

$f_{2}(r)=\alpha_{m}^{2} r^{2}-\frac{4 M}{\alpha_{g} r}-\alpha_{m}^{2} c_{1} r+\alpha_{m}^{2} c_{0}$,

where

$\alpha_{m}^{2} \equiv m_{g}^{2}(1+\alpha+\beta), \quad c_{1} \equiv \frac{h_{0}(1+2 \alpha+3 \beta)}{1+\alpha+\beta}$,

$c_{0} \equiv \frac{h_{0}^{2}(\alpha+3 \beta)}{1+\alpha+\beta}$.

The solution in Eq. (31) is an exact black string solution in dRGT massive gravity which, in the limit $\alpha_{m}=\alpha_{g}$ and $c_{0}=c_{1}=0$, naturally goes over to Lemos' [39] black string in general relativity. Henceforth, for definiteness, we shall call this massive gravity solution (31) a dRGT black string. In particular, it incorporates the cosmological-constant term naturally in terms of the graviton mass $m_{g}$, which should not be surprising since the graviton mass serves as the cosmological constant in the self-expanding cosmological solution in massive gravity. The graviton mass also generates a constant term which is known as the so-called global monopole term.

It is important to note that the strong coupling scale of the dRGT massive gravity theory is $\Lambda_{3}=\left(m_{g}^{2} M_{P l}\right)^{1 / 3}$. This is fixed by the construction of the mass terms of the theory [45], which may not be obvious from the action in Eq. (2). However, this scale can be simply seen through this solution. From this solution, the nonlinear scale known as the Vainshtein radius can be written as

$r_{V}=\left(\frac{M}{\alpha_{g} m_{g}^{2}}\right)^{1 / 3}=\left(\frac{M}{\alpha_{g} M_{P l}}\right)^{1 / 3} \frac{1}{\Lambda_{3}}$.

The parameter $M$ is dimensionful with mass per unit length scaled by $\alpha_{g}^{-1}$. Therefore, $M / \alpha_{g}$ is the mass of the black string. By taking the values within the solar system and $m_{g} \sim H$, one found that $r_{V} \sim 10^{16} \mathrm{~km} \gg \Lambda_{3}^{-1} \sim 10^{3} \mathrm{~km}$. In particular, this indicates that we do not have to worry about the strong coupling issue in dRGT massive gravity for a system of scale below $\Lambda_{3}$ (or of length scale beyond $\sim 10^{3} \mathrm{~km}$ ). The same situation also occurs in the spherically symmetric case [45].

One can see that the horizon structure depends on the sign of $\alpha_{m}^{2}$. If $\alpha_{m}^{2}>0$, corresponding to an anti-de Sitter-like solution, the maximum number of horizons is three. If $\alpha_{m}^{2}<0$, corresponding to de Sitter-like solution, the maximum number of horizons is two. The generic behavior of the horizon structure is shown in Fig. 1. This solution modifies the black string solution in general relativity with cosmological constant [39] by the three parameters $c_{0}, c_{1}$ and $\alpha_{m}^{2}$.

\subsection{Thermodynamics of dRGT black string}

Now, we analyze the thermodynamics of the new dRGT black string (31). By definition, the horizons are zeros of $f\left(r_{+}\right)=$ 0 . Depending on the parameters, there may exist up to three 
real roots. The horizon radius $\left(r_{+}\right)$and black string mass $\left(M_{+}\right)$are related via

$M_{+}=\frac{1}{4} \alpha_{g} \alpha_{m}^{2} r_{+}\left(r_{+}^{2}-c_{1} r_{+}+c_{0}\right)$.

The mass $M_{+}$depends on the parameters $c_{0}, c_{1}, \alpha_{m}^{2}$ and $\alpha_{g}^{2}$. To have a positive definite mass, one may require the term $\alpha_{m}^{2} r_{+}\left(r_{+}^{2}-c_{1} r_{+}+c_{0}\right)$ to be positive. Such a consideration yields the following conditions:

$\alpha_{m}^{2}>0, \quad c_{1}^{2}-4 c_{0}<0$.

The second condition is obtained from the requirement that $r_{+}^{2}-c_{1} r_{+}+c_{0}$ cannot be factorized so that, including the condition $\alpha_{m}^{2}>0$, the term $\alpha_{m}^{2} r_{+}\left(r_{+}^{2}-c_{1} r_{+}+c_{0}\right)$ will be positive definite. The Hawking temperature of the dRGT black string can be obtained by using $T=\kappa /(2 \pi)$, where $\kappa=f^{\prime}(r) /\left.2\right|_{r=r_{+}}$is the surface gravity of the black string. As a result, the temperature can be written as

$T_{+}=\frac{\alpha_{m}^{2}}{4 \pi r_{+}}\left(3 r_{+}^{2}-2 c_{1} r_{+}+c_{0}\right)$.

We can determine a condition for a positive definite temperature by using the same procedure as we did for the mass. Consequently, we obtain

$c_{1}^{2}-3 c_{0}<0$.

These positivity conditions for both the mass and the temperature can narrow down the parameter space. The minimum temperature can be found at $r_{+}=\sqrt{c_{0} / 3}$. At this point, the temperature can be written as

$T_{\min }=\frac{\alpha_{m}^{2}}{2 \pi}\left(\sqrt{3 c_{0}}-c_{1}\right)$.

The square root of $c_{0}$ is well defined through the conditions in Eq. (35) and/or in Eq. (37). Next, we can find its entropy via the first law of thermodynamics, $\mathrm{d} M_{+}=T_{+} \mathrm{d} S$; it reads

$S=\frac{1}{2} \pi \alpha_{g} r_{+}^{2}=\frac{A}{4}$,

$A=2 \pi \alpha_{g} r_{+}^{2}$,

and it turns out to satisfy the area law. Now we find the thermodynamical stability of the black string. For the local thermodynamical stability, we find the heat capacity of the system, which can be expressed as

$C=\left(T \frac{\mathrm{d} S}{\mathrm{~d} T}\right)_{r=r_{+}}=\frac{\pi \alpha_{g} r_{+}^{2}\left(3 r_{+}^{2}-2 c_{1} r_{+}+c_{0}\right)}{3 r_{+}^{2}-c_{0}}$.

There exists a minimum temperature; $C$ diverges at that temperature, where the corresponding radius obeys $r_{+}=\sqrt{c_{0} / 3}$. Moreover, the heat capacity is negative when $r_{+}<\sqrt{c_{0} / 3}$ and positive when $r_{+}>\sqrt{c_{0} / 3}$. This suggests that the local thermodynamical stability of the black string requires the condition $r_{+}>\sqrt{c_{0} / 3}$. Apart from the local one, the global thermodynamical stability can be analyzed by considering the Helmholtz potential

$$
\begin{aligned}
F & =M-T S \\
& =\frac{\alpha_{g} \alpha_{m}^{2} r_{+}}{8}\left(c_{0}-r_{+}^{2}\right) .
\end{aligned}
$$

The black string will be stable if the Helmholtz potential is negative. Thus, the global thermodynamical stability condition can be expressed as

$r_{+}>r_{c}=\sqrt{c_{0}}$.

Note that this condition also satisfies the local stability condition. As a result, the Hawking-Page phase transition, the transition from the hot flat space state to the black hole or black string state [46], occurs at the critical point, $r_{+}=r_{c}=\sqrt{c_{0}}$. It is interesting to note that the phase transition can be realized only for the asymptotic AdS solution. Moreover, the existence of the phase transition depends only on the graviton mass. This can be seen clearly when we consider the case $c_{1}=c_{0}=0$. In this case, the Helmholtz potential does not change sign, which means that the Lemos black string is always thermodynamically stable.

\section{Charged dRGT black string}

Even though the astronomical objects are neutral, the charged black holes are intensively investigated. For spherically symmetric solutions, the charged black hole is a useful toy model since the structure of the horizon usually differs that of the neutral one in a quite drastic way. Moreover, study of thermodynamics of the charged black holes can be significantly extended, since it usually involves an open system such as a grand canonical system where the charge can play the role of the chemical potential. In this section, we extend our consideration by adding the electric charge to the theory. As a result, the action can be obtained by adding a gauge field term as follows:

$S=\int \mathrm{d}^{4} x \sqrt{-g}\left[\frac{1}{2}\left[R+m_{g}^{2} \mathcal{U}\left(g, \phi^{a}\right)\right]-\frac{1}{16 \pi} F_{\mu \nu} F^{\mu \nu}\right]$,

where $F_{\mu \nu} \equiv\left(\nabla_{\mu} A_{\nu}-\nabla_{\nu} A_{\mu}\right), A_{\mu}=(a(r), 0,0,0)$ and $a(r)$ is an arbitrary function. By using the same procedure as in the non-charged case, the solutions still can be divided into two branches according to Eqs. (25) and (26). The equations of motion of the electric field, the Maxwell equations, provide the constraint to the function $a(r)$ of 
$a(r)=-\frac{\gamma}{\alpha_{g} r}$,

where $\gamma$ is an integration constant. In order to reduce the theory to the electromagnetic theory in flat spacetime, the integration constant can be written in terms of the linear charge density, $q$, in the $z$ direction as $\gamma^{2}=4 q^{2}$. By solving the modified Einstein equations, the solutions for both branches can be written as

$$
\begin{aligned}
f_{1}^{(q)}(r)= & \left(-\frac{m_{g}^{2}\left(1+\alpha+\alpha^{2}-3 \beta\right)}{3(\alpha+3 \beta)}\right) r^{2} \\
& -\frac{b}{\alpha_{g} r}+\frac{\gamma^{2}}{\alpha_{g}^{2} r^{2}}, \\
f_{2}^{(q)}(r)= & \frac{\gamma^{2}}{\alpha_{g}^{2} r^{2}}-\frac{b}{\alpha_{g} r}+m_{g}^{2}\left((1+\alpha+\beta) r^{2}\right. \\
& \left.-h_{0}(1+2 \alpha+3 \beta) r+h_{0}^{2}(\alpha+3 \beta)\right) .
\end{aligned}
$$

As we have mentioned before, we will focus on the second branch of the solutions which can be written in terms of the parameters in Eq. (32) as

$f(r)=\alpha_{m}^{2} r^{2}-\frac{4 M}{\alpha_{g} r}+\frac{4 q^{2}}{\alpha_{g}^{2} r^{2}}-\alpha_{m}^{2} c_{1} r+\alpha_{m}^{2} c_{0}$.

The solution (49) is the charged dRGT black string solution. The linear mass density $M$ can be expressed by solving $f\left(r_{+}\right)=0$ as follows:

$M^{(q)}=\frac{1}{4} \alpha_{g} \alpha_{m}^{2} r_{+}\left(r_{+}^{2}-c_{1} r_{+}+c_{0}\right)+\frac{q^{2}}{\alpha_{g} r_{+}}$.

For a charged dRGT black string, it can be treated as two kinds of thermodynamical systems; grand canonical and canonical systems. We will separate our considerations into the two following subsections.

\subsection{Grand canonical black string}

In this aspect of treatment, a charged dRGT black string is considered to be an open system where charge transfer or creation/annihilation are allowed. The energy gain/loss from the increase/decrease of charge is determined by the chemical potential,

$$
\mu=\frac{2 q}{\alpha_{g} r}
$$

evaluating at the horizon. The charged dRGT black string mass and the Hawking temperature can be written as

$$
\begin{aligned}
M_{G}^{(q)} & =\frac{1}{4} \alpha_{g} \alpha_{m}^{2} r_{+}\left(r_{+}^{2}-c_{1} r_{+}+c_{0}\right)+\frac{1}{4} \alpha_{g} r_{+} \mu^{2}, \\
T_{G}^{(q)} & =\frac{\alpha_{m}^{2}\left(3 r_{+}^{2}-2 c_{1} r_{+}+c_{0}\right)-\mu^{2}}{4 \pi r_{+}} .
\end{aligned}
$$

The positive definite of both mass and temperature can be found by following the same step as done in the non-charge case. As a result, the condition can be expressed as

$\alpha_{m}^{2}>0, \quad c_{1}^{2}-3\left(c_{0}-\frac{\mu^{2}}{\alpha_{m}^{2}}\right)<0$.

From these thermodynamic quantities, one found that they satisfy the first law of thermodynamics and the area law in which the entropy of the charged dRGT black string in the grand canonical system can be written as

$S_{G}^{(q)}=\frac{1}{2} \pi \alpha_{g} r_{+}^{2}=\frac{A}{4}$.

For the thermodynamical stability of the system, one can proceed finding the heat capacity and the Gibbs potential. By following the same strategy as done in the non-charge case, the heat capacity and the Gibbs potential can be found as

$C_{G}^{(q)}=\left(T \frac{\mathrm{d} S}{\mathrm{~d} T}\right)_{r=r_{+}}=\frac{\pi \alpha_{g} r_{+}^{2}\left[\alpha_{m}^{2}\left(3 r_{+}^{2}-2 c_{1} r_{+}+c_{0}\right)-\mu^{2}\right]}{\alpha_{m}^{2}\left(3 r_{+}^{2}-c_{0}\right)+\mu^{2}}$,

$G^{(q)}=M-T S-\mu Q=-\frac{\alpha_{g} r_{+}}{8}\left[\alpha_{m}^{2}\left(r_{+}^{2}-c_{0}\right)+\mu^{2}\right]$.

The thermodynamical stability conditions can be found by requiring $C_{G}^{(q)}>0$ and $G^{(q)}<0$. The resulting condition can be expressed as

$r_{+}>r_{c}=\sqrt{\left(c_{0}-\frac{\mu^{2}}{\alpha_{m}^{2}}\right)}$.

This suggests that it is possible to obtain the HawkingPage phase transition for charged dRGT black string in massive gravity theory, when considered in the grand canonical aspect. Note that this phase transition cannot be provided by using a Lemos black string solution. This issue can be seen by setting $c_{1}=c_{0}=0$, from which it turns out that the Gibbs potential is always negative for AdS solution while the dS solution does not satisfy the requirement of positive definite of the charged dRGT black string mass and temperature.

\subsection{Canonical black string}

For the canonical aspect of the black string, charge transfer or charge creation/annihilation are not allowed. The Hawking temperature can be found as

$T_{C}^{(q)}=\frac{1}{4 \pi r_{+}}\left[\alpha_{m}^{2}\left(3 r_{+}^{2}-2 c_{1} r_{+}+c_{0}\right)-\frac{4 q^{2}}{\alpha_{g}^{2} r_{+}^{2}}\right]$.

From this expression, we found that one of requirements to obtain the positive definite of the black string temperature is 
$\alpha_{m}^{2}>0$. This corresponds to the asymptotic AdS solution. By following the strategy as done before, we found that the system still obeys the first law of thermodynamics and the area law in which the entropy of the black string can be written as

$S_{C}^{(q)}=\frac{1}{2} \pi \alpha_{g} r_{+}^{2}=\frac{A}{4}$.

For the stability issue of this thermodynamic system, the local stability can be analyzed by considering the heat capacity which can be found as

$C_{C}^{(q)}=\frac{\pi \alpha_{g} r_{+}^{2}\left[\alpha_{m}^{2}\left(3 r_{+}^{2}-2 c_{1} r_{+}+c_{0}\right)-\frac{4 q^{2}}{\alpha_{g}^{2} r_{+}^{2}}\right]}{\alpha_{m}^{2}\left(3 r_{+}^{2}-c_{0}\right)+\frac{12 q^{2}}{\alpha_{g}^{2} r_{+}^{2}}}$.

By using $T_{C}^{(q)}>0$, the positivity of the heat capacity will be guaranteed by requiring

$r_{+}>\sqrt{\frac{c_{0}}{6}\left(1+\left[1-\frac{144 q^{2}}{\alpha_{g}^{2} \alpha_{m}^{2} c_{0}^{2}}\right]^{1 / 2}\right)}$.

The Helmholtz potential of the system can be written as

$F^{(q)}=-\frac{\alpha_{g} r_{+}}{8}\left[\alpha_{m}^{2}\left(r_{+}^{2}-c_{0}\right)-\frac{12 q^{2}}{\alpha_{g}^{2} r_{+}^{2}}\right]$.

To investigate the global thermodynamical stability, one requires that $F^{(q)}<0$. The condition turns out to be as follows:

$r_{+}>r_{c}=\sqrt{c_{0}\left(1+\left[1+\frac{144 q^{2}}{\alpha_{g}^{2} \alpha_{m}^{2} c_{0}^{2}}\right]^{1 / 2}\right)}$,

which is stronger than the one for the local stability condition. This suggests that, for the canonical system, it is possible to provide the Hawking-Page phase transition for the charged black string in massive gravity theory. However, for this system, the AdS black string solution can also provide the phase transition. This can be seen by setting $c_{1}=c_{0}=0$, from which it turns out that the phase transition occurs when $r_{+}^{2}=\sqrt{\frac{2 q}{\sqrt{3 \alpha_{m}^{2} \alpha_{g}^{2}}}}$.

\section{Concluding remarks}

The dRGT massive gravity describes nonlinear interaction terms as a generalization of the Einstein-Hilbert action when the graviton is massive. It is believed that dRGT massive gravity may provide a possible explanation for the accelerating expansion of the universe that does not require any dark energy or cosmological constant. In this paper, we have presented a class of black strings, both charged and uncharged, in dRGT massive gravity where the effective cosmological constant is negative, and we studied the thermodynamics and phase structure of the solutions in both the grand canonical and the canonical ensembles. The black string obtained is immensely simplified due to the choice of the fiducial metric. As expected the solution contains the Lemos solutions [39] as a particular case. Our dRGT black string solution can be identified, e.g., as a monopole-black string of general relativity for a suitable choice of the parameters of the theory where the graviton mass in massive gravity naturally generates the cosmological constant and the global monopole term.

We have also analyzed the thermodynamical properties of the dRGT black string solution. The thermodynamic quantities have also been found to contain corrections from the graviton mass except for the black string entropy which is unaffected by massive gravity and still obeys the area law. By analyzing the thermodynamical properties of the black string solution, we found that it is possible to obtain the HawkingPage phase transition in the dRGT black string case, while this is not possible for Lemos solution. The conditions to provide such a transition were explored. In fact, we determined the phase transition in both charged and non-charged case by analyzing the sign of the potential at $r=r_{c}$, with the stable (unstable) branch for $r>(<) r_{c}$.

The results presented here are a generalization of previous discussions of Lemos [39-41], on the black string, in a more general setting. The possibility of a further generalization of these results to higher dimensions is an interesting problem for future research. The rotating dRGT black string solution is also interesting, since most astronomical objects are rotating. We leave this investigation for further work.

Acknowledgements This project is supported by the ICTP through Grant no. OEA-NT-01 and the National Research Foundation of Korea (NRF) Grant funded by the Korea government (MSIP) (no. 2016R1C1B1010107). PW is also supported by the Naresuan University Research Fund through Grant no. R2559C235. S.G.G. would like to thank SERB-DST Research Project Grant no. SB/S2/HEP-008/2014 and also IUCAA, Pune, where part of this work was done, for hospitality.

Open Access This article is distributed under the terms of the Creative Commons Attribution 4.0 International License (http://creativecomm ons.org/licenses/by/4.0/), which permits unrestricted use, distribution, and reproduction in any medium, provided you give appropriate credit to the original author(s) and the source, provide a link to the Creative Commons license, and indicate if changes were made. Funded by SCOAP . $^{3}$

\section{References}

1. D.G. Boulware, S. Deser, Phys. Rev. D 6, 3368 (1972) 
2. C. de Rham, G. Gabadadze, Phys. Rev. D 82, 044020 (2010). arXiv:1007.0443 [hep-th]

3. C. de Rham, G. Gabadadze, A.J. Tolley, Phys. Rev. Lett. 106 , 231101 (2011). arXiv:1011.1232

4. D. Vegh, arXiv:1301.0537 [hep-th]

5. R.G. Cai, Y.P. Hu, Q.Y. Pan, Y.L. Zhang, Phys. Rev. D 91, 024032 (2015). arXiv:1409.2369 [hep-th]

6. S.G. Ghosh, L. Tannukij, P. Wongjun, Eur. Phys. J. C 76(3), 119 (2016). arXiv:1506.07119 [gr-qc]

7. A. Adams, D.A. Roberts, O. Saremi, Phys. Rev. D 91(4), 046003 (2015). arXiv:1408.6560 [hep-th]

8. J. Xu, L.M. Cao, Y.P. Hu, Phys. Rev. D 91, 124033 (2015). arXiv: 1506.03578 [gr-qc]

9. T.M. Nieuwenhuizen, Phys. Rev. D 84, 024038 (2011). arXiv:1103.5912 [gr-qc]

10. R. Brito, V. Cardoso, P. Pani, Phys. Rev. D 88, 064006 (2013). arXiv:1309.0818 [gr-qc]

11. L. Berezhiani, G. Chkareuli, C. de Rham, G. Gabadadze, A.J. Tolley, Phys. Rev. D 85, 044024 (2012). arXiv:1111.3613 [hep-th]

12. Y.F. Cai, D.A. Easson, C. Gao, E.N. Saridakis, Phys. Rev. D 87, 064001 (2013). arXiv:1211.0563 [hep-th]

13. E. Babichev, A. Fabbri, JHEP 1407, 016 (2014). arXiv:1405.0581 [gr-qc]

14. M.S. Volkov, Class. Quantum Gravity 30, 184009 (2013). arXiv:1304.0238 [hep-th]

15. G. Tasinato, K. Koyama, G. Niz, Class. Quantum Gravity 30, 184002 (2013). arXiv:1304.0601 [hep-th]

16. E. Babichev, R. Brito, Class. Quantum Gravity 32, 154001 (2015). arXiv:1503.07529 [gr-qc]

17. F. Capela, P.G. Tinyakov, JHEP 1104, 042 (2011). arXiv: 1102.0479 [gr-qc]

18. E. Babichev, C. Deffayet, Class. Quantum Gravity 30, 184001 (2013). arXiv:1304.7240 [gr-qc]

19. A. Salam, J.A. Strathdee, Phys. Rev. D 16, 2668 (1977)

20. C.J. Isham, D. Storey, Phys. Rev. D 18, 1047 (1978)

21. D. Comelli, M. Crisostomi, F. Nesti, L. Pilo, Phys. Rev. D 85, 024044 (2012). arXiv:1110.4967 [hep-th]

22. M.S. Volkov, Phys. Rev. D 85, 124043 (2012). arXiv:1202.6682 [hep-th]
23. Z. Berezhiani, D. Comelli, F. Nesti, L. Pilo, JHEP 0807, 130 (2008). arXiv:0803.1687 [hep-th]

24. K. Koyama, G. Niz, G. Tasinato, Phys. Rev. D 84, 064033 (2011). arXiv:1104.2143 [hep-th]

25. K. Koyama, G. Niz, G. Tasinato, Phys. Rev. Lett. 107, 131101 (2011). arXiv:1103.4708 [hep-th]

26. F. Sbisa, G. Niz, K. Koyama, G. Tasinato, Phys. Rev. D 86, 024033 (2012). arXiv:1204.1193 [hep-th]

27. Y.P. Hu, X.M. Wu, H. Zhang, arXiv:1611.09042 [gr-qc]

28. Y.P. Hu, X.X. Zeng, H.Q. Zhang, Phys. Lett. B 765, 120 (2017). arXiv:1611.00677 [hep-th]

29. D.C. Zou, R. Yue, M. Zhang, arXiv:1612.08056 [gr-qc]

30. S.H. Hendi, R.B. Mann, S. Panahiyan, B.E. Panah, Phys. Rev. D $\mathbf{9 5}(2), 021501(2017)$

31. S.H. Hendi, G.H. Bordbar, B.E. Panah, S. Panahiyan, arXiv:1701.01039 [gr-qc]

32. S.H. Hendi, B.E. Panah, S. Panahiyan, M.S. Talezadeh, arXiv: 1612.00721 [hep-th]

33. B.E. Panah, S. Panahiyan, arXiv:1611.10151 [hep-th]

34. S.H. Hendi, S. Panahiyan, S. Upadhyay, B.E. Panah, arXiv:1611.02937 [hep-th]

35. S.H. Hendi, N. Riazi, S. Panahiyan, arXiv:1610.01505 [hep-th]

36. S.H. Hendi, G.Q. Li, J.X. Mo, S. Panahiyan, B. Eslam, Panah. Eur. Phys. J. C 76(10), 571 (2016). arXiv:1608.03148 [gr-qc]

37. K.S. Thorne, in Magic without magic, ed. J. R. Klander (Freeman, San Francisco, 1972)

38. J.P.S. Lemos, Phys. Rev. D 59, 044020 (1999)

39. J.P.S. Lemos, Class. Quantum Gravity 12, 1081 (1995)

40. J.P.S. Lemos, Phys. Lett. B 353, 46 (1995). [gr-qc/9404041]

41. N.O. Santos, Class. Quantum Gravity 10, 2401 (1993)

42. J.P.S. Lemos, V.T. Zanchin, Phys. Rev. D 54, 3840 (1996)

43. R.G. Cai, Y.Z. Zhang, Phys. Rev. D 54, 4891 (1996). [grqc/9609065]

44. T. Chullaphan, L. Tannukij, P. Wongjun, JHEP 06, 038 (2015). arXiv: 1502.08018 [gr-qc]

45. K. Hinterbichler, Rev. Mod. Phys. 84, 671 (2012). arXiv:1105.3735 [hep-th]

46. S.W. Hawking, D.N. Page, Commun. Math. Phys. 87, 577 (1983) 